\title{
Sistemas de Recomendaciones: Una herramienta para mejorar la gestión de la información en las PYMES
}

\section{Systems of Recommendations: A tool for improve information management in SMEs}

\author{
Gladys Guevara Albán ${ }^{1, *}$, Cristian Guevara Albán $^{2, \dagger}$, e Ivette Valverde $2, \ddagger$ \\ ${ }^{1}$ Universidad Técnica de Babahoyo, Ecuador. \\ ${ }^{2}$ Instituto Tecnológico Superior Babahoyo, Ecuador. \\ gguevara@utb.edu.ec; \{cguevara;ivalverde\}@institutobabahoyo.edu.ec
}

Fecha de recepción: 15 de agosto de 2017 — Fecha de aceptación: 15 de septiembre de 2017

\begin{abstract}
Cómo citar: Guevara Albán, G., Guevara Albán, C., \& Valverde, I. (2018). Sistemas de Recomendaciones: Una herramienta para mejorar la gestión de la información en las PYMES. Journal of Science and Research: Revista Ciencia e Investigación, 3(CITT2017), 121-127. https://doi.org/10.26910/issn.2528-8083vol3issCITT2017.2018pp121-127
\end{abstract}

\begin{abstract}
Resumen-La aparición de los sitios web, el crecimiento de las redes sociales, la búsqueda de formas más eficiente de recaudar información y analizarlas para la toma decisiones en tiempo real y la forma de como las personas se agrupan y planean consumir productos y/o servicios como parte de una actividad conjunta nacen los sistemas de recomendadores(SR) siendo este objeto de nuestro estudio y cuyo objetivo principal es incrementar el nivel de confianza en la gestión de la información y la toma de decisiones en las PYMES aplicando SR. La línea de investigación en recomendadores, que se encuentra actualmente en auge, sufre una serie de deficiencias, radicadas en un incorrecto modelado de los comportamientos sociales de los usuarios, que reducen su eficiencia. El presente estudio es de tipo documental, descriptivo y de campo. En cuanto a las técnicas se aplicó revisión documental, cuestionario y la entrevista para así tener una noción global del espectro de estudio. En cuanto a los resultados obtenidos, microempresarios que son objeto de este estudio manifestaron la necesidad de disponer de un sitio que mejore la búsqueda ordenada de información por recomendaciones aplicando un modelo de búsqueda específico mejorado el proceso de la toma de decisiones y los servicios al cliente.
\end{abstract}

Palabras Clave-Sistemas de recomendaciones, Gestión de información, e-Commerce, PYMES.

\begin{abstract}
The appearance of websites, the growth of social networks, the search for forms more efficient to collect information and analyses them for making decisions in real time and the way people gather and plan to consume products and/or services. As part of a joint activity, the recommender (SR) systems are born this object of our study and whose main objective is to increase the level of confidence in the management of information and decision making in SMEs applying MR. The line of research on recommender, which is currently booming, suffers a series of deficiencies, rooted in an incorrect modeling of the social behaviors of users, which reduce their efficiency. The present study is a documentary, descriptive and field type. As for the techniques, a revision was applied documentary, questionnaire, and interview therefore as for having a global notion of the spectrum of study. Regarding the results obtained, micro-entrepreneurs who are the object of this study showed the need to have a site that improves the orderly search of information by recommendations applying a specific search model improved the process of decision making and customer services.
\end{abstract}

Keywords-Systems of recommendations, Information management, e-Commerce, SMEs.

\section{INTRODUCCIÓN}

$\mathbf{U}$ no de los principales problemas que existe en el Ecuador es que el comercio electrónico aún no se lo ha implementado y adoptado por completo, ya que existen dudas y falta de convicción por parte de los empresarios sobre la confiabilidad que existe en la venta de sus productos/servicios a través de la web. He aquí el objetivo de la presente investigación la cual es incrementar el nivel de confianza en los consumidores y productores en el uso del internet, para la venta de sus productos y servicios a través de website utilizando sistemas de recomendación(SR), permitiendo a las pequeñas y medianas empresas disponer de este tipo de sistemas sin riesgos de perdidas y sin invertir demasiado dinero y esfuerzos.

\footnotetext{
*Magister en Informática Educativa y Multimedios.

${ }^{\dagger}$ Ingeniero en Sistemas.

¥Ingeniera en Sistemas.
}

\section{LAS PYMES, MARKETING DIGITAL Y LA TECNOLOGÍA MÓVIL EN EL ECUADOR}

"Según estadísticas, el Ecuador mueve al año USD 200 millones en ventas en línea, según el Instituto Latinoamericano de Comercio Exterior. En cambio, las cifras del Instituto Nacional de Estadística y Censos (INEC) son superiores y señalan que en el país se generan USD 540 millones" (El Telégrafo, 2016).

En el Ecuador la mayor parte de la publicidad de los productos que ofertan las microempresas que se apoyan en el internet, es a través de redes sociales como Facebook, seguida por Twitter y Google. Rafael Campos, especialista en comercio electrónico, señaló en el E-Commerce Day (julio) El Comercio (2016), que un $60 \%$ de los paquetes que se distribuyen Correos del Ecuador son productos de Amazon.com. Observando así que el crecimiento de los consumidores en optar por la cultura de la compra en línea. "Jorge Rosales, delegado del Mipro, 
destaca que el e-commerce abre la posibilidad de generar transacciones en cualquier momento y lugar. "Al ser Guayaquil la capital comercial del país, es importante que se ponga al nivel tecnológico que requieren nuestros empresarios por sus beneficios". Expresa que unas 5.200 empresas, entre medianas y pequeñas, además de artesanos, reciben capacitación constante de acceso a la tecnología” (El Telégrafo, 2016).

Esto permitirá a futuro que se incremente la confianza de consumidores y productores en la venta por internet de sus productos. En los últimos años las PYMES han adquirido sistema de venta en línea, donde uno de los beneficios de ofrecer sus productos/servicios bajo este sistema es abaratar costos, pero que no están conformes con esto, ya que al no interactuar directamente con el consumidor y que no conocer sus gustos y características específicas, siente que no están dando un servicio de calidad.

Debido a la gran cantidad de información que se encuentran en Internet, a veces resulta difícil para los usuarios encontrar los contenidos que realmente necesitan de una manera fácil y rápida. Los usuarios tienden a buscar orientación en otras personas que previamente han tenido la misma necesidad; ó bien seleccionan aquellos objetos que más se parecen a lo que buscan (Sanjuan-Martinez et al., 2009).

Según ha señalado el director general de Rocket Fuel en España, Juan Sevillano Zabala, "el próximo año seremos testigos de la maduración del marketing digital, así como de un importante crecimiento. Crecerá la necesidad de tecnología en tiempo real y se extenderá el marketing predictivo"(Ekos, 2016).

De acuerdo a estadísticas dadas por ARCOTEL el Internet móvil refleja un incremento importante de las cuentas de este servicio desde 2010. En los dos últimos años, la tasa promedio de crecimiento en el servicio de internet móvil está directamente relacionada con la penetración del SMA y la implementación de la tecnología 3G y 4G, han facilitado el acceso a los usuarios a plataformas de navegación cuyo uso se encuentra incentivado por la oferta de contenidos y el acceso a las redes sociales (ARCOTEL, 2017).

En la actualidad el manejo de grandes volúmenes de información en bases de datos es sin estructurar en la red estos corresponden a: e-mail videos, tweets, comentarios en Facebook, circuito cerrado TV, chat en línea, llamadas móviles, msm, etc.

\section{SISTEMAS DE RECOMENDACIÓN}

Debido a los grandes volúmenes de información que se gestiona en la web, cada vez se hace más difícil al realizar una búsqueda de información. Los SR se han ido consolidando como potentes herramientas para ayudar a reducir la sobrecarga de información a la que nos enfrentamos en los procesos de búsqueda de información. Ayudan a filtrar los ítems de información recuperados, usando distintas técnicas para identificar aquellos ítems que mejor satisfacen las preferencias o necesidades de los usuarios. Las recomendaciones se generan a partir de las opiniones proporcionadas por otros usuarios sobre esos ítems en búsquedas previas, o bien a partir del perfil del usuario (Herrera-Viedna et al., 2004).
La recuperación de información en la web y los sistemas de recomendaciones permiten evaluar y filtrar la información usando recomendaciones propuestas a partir de opiniones dadas por los usuarios. Estas opiniones se convierten en ítems de búsqueda basándose a partir de los gustos o preferencias de los consumidores de los productos/servicios. Cabe destacar que estos "sistemas producen recomendaciones personalizadas como salida o tienen el efecto de guiar al usuario de una forma personalizada a productos interesantes o útiles de entre una gran cantidad de productos disponibles" (Robin, 2002).

Para que los SR alcance sus metas, necesita conocer los gustos y acciones explicitas de los usuarios en ámbito de compras, basado en las relaciones colectivas de los usuarios asociados en comunidades de consumidores de una red social, permite construir un conocimiento colectivo que ayude a recomendar de forma automática listas de contenidos a los usuarios de una plataforma social, basados en su comportamiento, preferencias y antecedentes de compra (López et al., 2011).

Un sistema "recomendador" permite al usuario seleccionar un producto/servicio, para su posterior compra, alimentando así la base de datos de la empresa con características específicas acerca de lo adquirido. Para hacer las recomendaciones, el sistema analiza y procesa información histórica de los usuarios (edad, compras previas, calificaciones...), de los productos o de los contenidos (marcas, modelos, precios, contenidos similares...) Carrillo Agurto (2017) y la transforma en conocimiento accionable, es decir, predice qué producto puede ser interesante para el usuario y para la empresa. Los recomendadores al momento de presentar la información al usuario final guarda un nivel de autonomía que solo lo rige las empresas al momento de presentarla en la web.

Por lo general los recomendadores más utilizados en el marketing digital son: los filtros colaborativos y los filtros basados en contenido (Galán Nieto, 2007).

Estos se basan en algoritmos matemáticos el cual "toma la decisión" de cual recomendación es la más acertada al momento de mostrar los datos. El filtrado colaborativo (Herlocker et al., 1999) consiste en aplicar algoritmos de acuerdo a las características de los usuarios a los que hay que realizarle las recomendaciones.

El rol más importante en este tipo de filtrado es recomendar aquellos ítems que no han sido votados por el usuario activo $\mathrm{y}$ que han resultado bien valorados por los usuarios similares. La lógica se basa en el análisis de las compras anteriores, las preferencias, las calificaciones que ha dado de otros productos, el importe medio de las compras, etc. y busca otros usuarios que se parecen a él y que han tomado decisiones parecidas. En cuanto al filtrado basados en contenido se fundamenta en el conocimiento que se tiene sobre el producto como base de la predicción, en lugar de tener en cuenta al usuario (Herlocker et al., 1999).

Recomendando ítems (Herlocker et al., 2004) similares que pueden gustar o interesar al usuario, como, por ejemplo: Sistema de recomendaciones para la compra de zapatos. En este caso el producto sería los Zapatos. Los datos que se recopilaría son: la marca, precio, modelo, color, tamaño, etc. Para alimentar al sistema de información se debe de tomar en cuenta las calificaciones tanto explicitas con el número de 
estrellas que le dan al producto o implícitas como el número de veces $q$ ha comprado zapatos de las mismas especificaciones. Además de los anteriores filtros de recomendaciones mencionados también se utilizan Filtrado Demográfico y los métodos de Filtrado Híbrido. En cuanto al demográfico hace referencia a las características de los usuarios (ciudad, profesión, sexo, edad, etc. Y el Filtrado Hibrido hace referencia a la mezcla de los tres filtrados mencionados anteriormente y en algunos de los casos combinan técnicas de inteligencia de negocios, inteligencia artificial, lógica borrosa, entre otros.

\section{CONSIDERACIONES A TOMAR EN CUENTA AL MOMENTO DE DISEÑAR UN RECOMENDADOR}

Al momento de diseñar un recomendador para una microempresa se debe de considerar, si manejan gran volumen de información, la cartera de clientes es extensa y el catálogo de productos es considerable, sino se cumple con esto, no es recomendable aplicar y sistema de recomendador. Luego se debe tomar en cuenta a que lugar el usuario tiene acceso para observar las recomendaciones, además se debe se analizar la ubicación y el momento adecuado de presentar la recomendación en la web, para que el usuario no se distraiga y pueda visualizarla (al ingreso, durante la visita, final o a los lados de la página). En cambio, en una website puede ser más eficiente situarlas en la parte inferior del producto que se está viendo y al final del proceso de compra. Se debería aplicar un testing como técnica para tomar este tipo de decisiones. Al momento de diseñar el SR se debe de tomar en cuenta el punto de vista del cliente y del negocio. En cuanto al primer aspecto a tomar en cuenta es mejor hacer recomendaciones arriesgadas que ofrezcan productos desconocidos para el cliente que una recomendación demasiado obvia que se corre el riesgo de no sea la más útil. Desde el punto de vista de negocios es necesario tener en cuenta la lógica de negocios para ajustar la rentabilidad del producto como parámetro de toma de decisiones. En la fase de ingeniería de datos, selección de características, categorización se deberá realizar un análisis masivo de datos para hacer predicciones (Sarwar et al., 2017).

\section{Metodología}

En el presente trabajo se realizó una investigación documental con respecto a los sistemas de recomendaciones y las tendencias del marketing electrónico, estadísticas de las pymes del Ecuador, donde los métodos de análisis y síntesis permitieron obtener una visión general de cómo articular mejorar la gestión de la información en las pymes a través de los SR. Se analizaron las estadísticas dadas por el instituto nacional ecuatoriano de censos en el último levantamiento de información dado en el 2015, sobre el crecimiento de las microempresas en el Ecuador, dando una idea global de que este sector micro empresarial están teniendo un crecimiento gigantesco en los últimos años. Además, se analizó las últimas tendencias referente a Tecnologías de la Información y Comunicaciones (TIC) y su impacto en los consumidores finales y el uso de la tecnología móvil y las redes sociales.

Se reviso el nivel de penetración entre los consumidores al Facebook hasta junio del 2016, en página de internet
World Stats, registrada por la American Registry for Internet Numbers(Group Miniwatts Marketing, 2017).

Se aplico entrevistas a expertos en marketing digital y miembros de empresas donde destacan la importancia de las oportunidades que ofrecen estas herramientas digitales en el ámbito publicitario, el manejo de grandes volúmenes de información que es el activo fijo de los microempresarios y hacia dónde va la tendencia de conocer las preferencias de los consumidores. Se entrevisto administradores de comunidades y programadores de website, tomando temas sobre como ofrecen sus clientes sus productos/servicios, y que tipo de tratamiento dan a la información y que métodos o algoritmos están aplicando para la toma de decisiones. Se selecciono una muestra de 25 microempresarios, donde se aplicó una encuesta con respecto a cómo: promocionan y venden sus productos/servicios, si poseen conocimientos en: venta digital, sistemas de recomendaciones en la web, predicciones y recomendaciones basadas en el feedback de otras personas, también se tomó en cuenta si ellos están predispuestos de invertir en la implementación de website para sus microempresas, ya que estas les proporciona información detallada y conocimiento específico sobre los perfiles demográficos y psicográficos de los clientes, y que pueden combinar con datos de terceros cuando sea necesario.

\section{RESULTADOS}

Se realizo un análisis de las microempresas y su actividad económica en el Ecuador, para sacar un conceso del futuro del marketing digital en las pymes, el uso del internet y telefonía móvil y la importancia del manejo de grandes volúmenes de información a través sistemas de recomendaciones. A continuación, se muestra varias estadísticas recogidas en los últimos cinco años, que sirvió como referente para este estudio. De acuerdo a los datos del Directorios de empresas en el Ecuador del 2017 INEC (2015), Figura 1, entre el 2012 hasta el 2015 se observa un incremento $15 \%$ en el aumento del número de microempresas en el Ecuador.

\begin{tabular}{|lrrrrr|}
\hline \multicolumn{5}{c|}{ Número de Empresas por Tamaño de Empresa } \\
\hline Tamaño de Empresa & 2012 & 2013 & 2014 & 2015 \\
Microempresa & & 656.406 & 731.073 & 760.472 & 764.001 \\
Pequeña empresa & & 59.399 & 61.785 & 65.330 & 61.987 \\
Mediana empresa A & 7.102 & 7.583 & 7.961 & 7.733 \\
Mediana empresa B & 4.862 & 5.244 & 5.632 & 5.156 \\
Grande empresa & & 3.591 & 3.896 & 4.228 & 4.059 \\
\hline
\end{tabular}

Figura 1. Número de empresas por tamaño de empresa.

Fuente: Ecuador en cifras.

En cuanto al número de empresas por tamaño de empresa y participación nacional registradas en el DIEE INEC (2015), en el año 2015. En la Figura 2, se observa que existe 90,6\% de microempresas siendo ampliamente mayoritarias en relación a las empresas pequeñas, medianas y grandes empresas que ocupan un porcentaje sumamente menor.

De acuerdo al Análisis y Ranking de PyMES de acuerdo a su actividad económica de las microempresas, Figura 3, siendo el 46,2 \% de las PYMES en nuestro país están concentradas 


\begin{tabular}{|l|r|r|}
\hline Tamaño de empresa & Nro. Empresas & \% Total \\
\hline TOTAL & $\mathbf{8 4 2 . 9 3 6}$ & $\mathbf{1 0 0 , 0 \%}$ \\
MICROEMPRESA & 764.001 & $90,6 \%$ \\
\hline PEQUENAA EMPRESA & 61.987 & $7,4 \%$ \\
\hline MEDIANA EMPRESA "A" & 7.733 & $0,9 \%$ \\
MEDIANA EMPRESA "B" & 5.156 & $0,6 \%$ \\
\hline GRANDE EMPRESA & 4.059 & $0,5 \%$ \\
\hline
\end{tabular}

Figura 2. Número de empresas por tamaño de empresa en participación nacional.

Fuente: DIEE.

en las actividades de manufactura y comercio, siendo ampliamente mayoritarias aquellas microempresas de esta última con el 37,6\% del total en el año 2015 INEC (2015) (dentro del sector comercial, tiene mayor peso las actividades, los establecimientos de alojamiento y restaurantes con el $8 \%$ ). Otro sector de localización importante para las PYMES, con el $20 \%$ de participación, es el de "Finanzas, seguros, transporte y almacenamiento, construcción y servicios profesionales", en el cual se agrupan gran cantidad de empresas que brindan diferentes servicios.

\begin{tabular}{|c|c|}
\hline Actividad Económica & Microempresa \\
\hline COMERCIO, REPARACIÓN AUTOMOTORES Y MOTOCICLETAS & $37,6 \%$ \\
\hline TRANSPORTE Y ALMACENAMIENTO & $11,0 \%$ \\
\hline AGRICULTURA, GANADERIA, SILVICULTURAY Y PESCA & $11,0 \%$ \\
\hline INDUSTRIAS MANUFACTURERAS & $8,6 \%$ \\
\hline ACTIVIDADES DE ALOJAMIENTO Y DE SERVICIO DE COMIDAS & 8,036 \\
\hline ACTIVIDADES PRDFESIONALES, CIENTIFICAS Y TÉCNICAS & $6,2 \%$ \\
\hline DTRAS ACTIVIDADES DE SERVICIOS & $4,7 \%$ \\
\hline CONSTRUCCIÓN & $3,3 \%$ \\
\hline ACTIVIDADES INMOBILIARIAS & $2,4 \%$ \\
\hline ACTIVIDADES DE SERVICIOS ADMINISTRATIVOS Y DE APOYO & $2,1 \%$ \\
\hline ACTIVIDADES DE ATENCIÓN A LA SALUD HUMANA Y ASISTENCIA SOCINL. & $2,1 \%$ \\
\hline ENSEÑANZA & $1,1 \%$ \\
\hline ARTES, ENTRETENIMIENTO Y RECREACIÓN & $0,8 \%$ \\
\hline INFORMACIÓN Y COMUNICACIÓN & $0,5 \%$ \\
\hline EXPLOTACIÓN MINAS Y CANTERAS & $0,4 \%$ \\
\hline ACTIVIDADES FINANCIERAS Y DE SEGUROS & $0,2 \%$ \\
\hline ADMINISTRACIÓN PÚBLICA Y DEFENSA, SEGURIDAD SOCIAL & $0,1 \%$ \\
\hline DISTRIBUCIÓN AGUA; ALCANTARILADO, DESECHOS Y SANEAMIENTO & $0,1 \%$ \\
\hline SUMINISTRO ELECTRICIDAD, GAS, VAPOR Y AIRE ACONOICIONADO & $0,0 \%$ \\
\hline
\end{tabular}

Figura 3. Porcentaje de empresas según tamaño y actividad económica.

Fuente: DIEE.

Hasta marzo de 2017, en Ecuador con una población de 16'625,776 habitantes, se observa que 13'471,736 usuarios usan internet, son el $81 \%$ de nivel de penetración entre los consumidores según la página de internet World Stats, Facebook registraba 9'700.000 usuarios que hicieron ingresaron con el 58,3\% (Group Miniwatts Marketing, 2017).

ARCOTEL (IAB, 2014) manifiesta que en 46,4\% de usuarios del Servicio Móvil Avanzado poseen un smartphone debido al alto consumo de internet Figura 4.

"Un estudio de Hábitos Digitales en Ecuador, realizado entre mayo y junio de 2014 por medio de entrevistas a 1.134 personas, a través de un panel de internautas, revela que

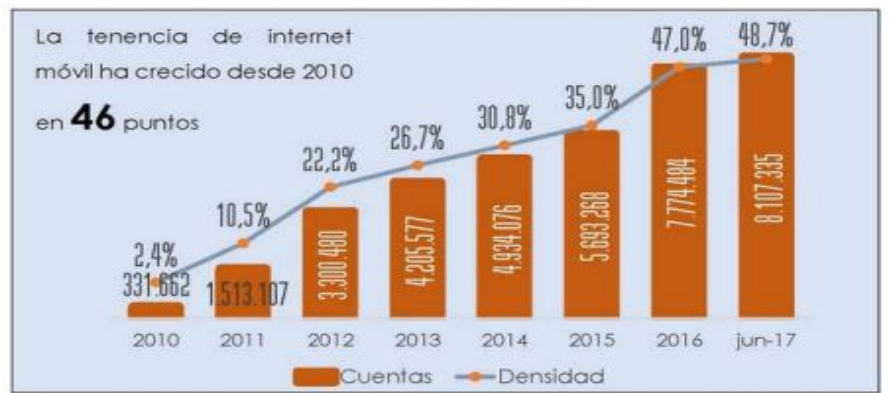

Figura 4. Tendencia del Internet móvil en porcentajes.

Fuente: ARCOTEL.

un $97 \%$ de ellas estaba en redes sociales, según el análisis publicado por Interactive Advertising Bureau (IAB) Ecuador, que era filial de un organismo mundial que representa y fomenta la publicidad en internet. La preferida era Facebook, seguida por Twitter y Google+" (IAB, 2014).

Luego en la presente investigación se aplicaron técnicas de observación, encuestas, entrevistas a varios actores relacionados con el e-commerce y microempresarios a continuación, se detallan: Se entrevistaron a experto en marketing digital y administradores de comunidades independientes, donde opinan que en los últimos cuatro años se han incrementado su trabajo, ante el boom de cliente que optan por publicitar sus productos/servicios a través de redes sociales. Además, recomiendan a sus clientes en que deben de tener su propia website, ya que será su carta de presentación, podrán contar con todos datos y detalles del producto que promocionan y que con la aplicación de métodos de filtrado podrán conocer los gustos y tendencia de sus consumidores y así poder predecir y tomar decisiones optimas al momento de vender. En cuanto a las entrevistas realizadas con programadores de website, manifestaron que en la actualidad se da mucho énfasis a la calidad de la información, al momento de escoger el método o algoritmos para el filtrado de la información. Afirman que, con datos de calidad, una buena base de datos que maneje gran cantidad de información de manera organizadas, se brindara buenas recomendaciones. Se está utilizando en un $89 \%$ el método de filtrado mixto, el cual está facilitando el análisis masivo de datos para hacer predicciones futuras tomando en cuenta los filtrados demográficos, colaborativos y de contenidos. Que en los últimos años los microempresarios están adquiriendo esta herramienta digital pero que aún se les hace difícil al momento de pactar costos para la creación de su propio website. De acuerdo a las entrevistas y a la muestra de la población encuestas aplicadas de micro empresarios, se clasificaron en tres grupos (Figura 5): el 56\% de los microempresarios realizan sus ventas en línea, donde expresan que en los últimos tres años han observado un incremento considerable en ventas, de este grupo el $58 \%$ publicitan sus productos a través de Facebook, seguido por WhatsApp y Twitter, el $22 \%$ lo realizan a través de un website(Fig. 7.); además manifestaron que el volumen de información que maneja es extensa. En cuanto a que si utilizan SR manifestaron un $65 \%$ que no tienen conocimiento sobre este tema, que lo han aplicado de manera 
empíricamente, aunque sin saberlo estos datos les ayudado a predecir y a la toma de decisiones, el $28 \%$ han contratado a expertos en marketing digital.

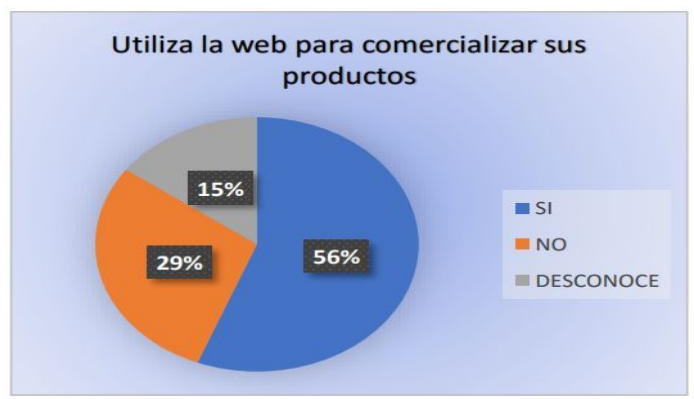

Figura 5. Porcentaje de comercialización de productos/servicios en la Web por microempresarios.

Fuente: Elaboración Propia.

El $29 \%$ del segundo grupo previamente clasificado, opinan que aún no adoptan esta tecnología de venta on line, aunque si tienen conocimiento que a través de redes sociales lo pueden realizar (Figura 6), manifiestan que el volumen de información que manejan es considerable para adoptar esta la tecnología de sistema de recomendaciones, y que a futuro van a publicitar sus productos a través de redes sociales y la creación de un website propio para ofertar sus productos (Figura 7).

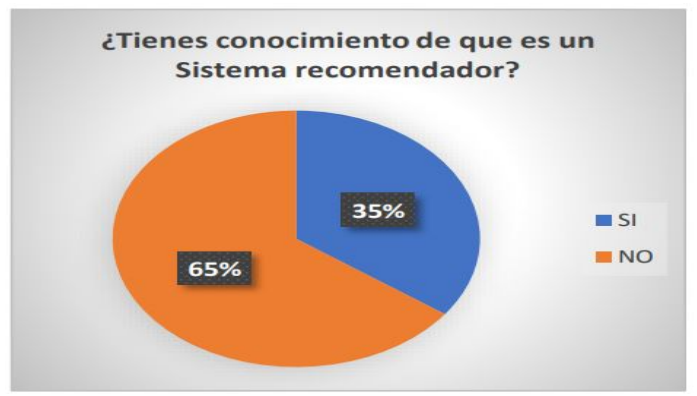

Figura 6. Porcentaje de conocimiento de que es un sistema Recomendador por los microempresarios

Fuente: Elaboración Propia.

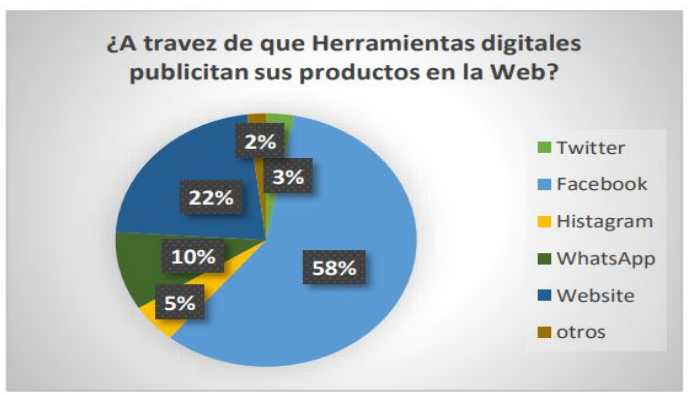

Figura 7. Porcentaje de uso de herramientas digitales publicitan sus productos en la Web.

Fuente: Elaboración Propia.

El tercer grupo que representa el $15 \%$, no tienen idea de que es marketing digital, y de este grupo el $85 \%$ si tienen acceso a redes sociales y han observado como otros microempresarios y empresas nacionales y multinacionales publicitan sus productos/servicios, que les gustaría publicitar sus productos y les parece novedoso poder predecir y conocer los gustos de sus clientes (Figura 8).

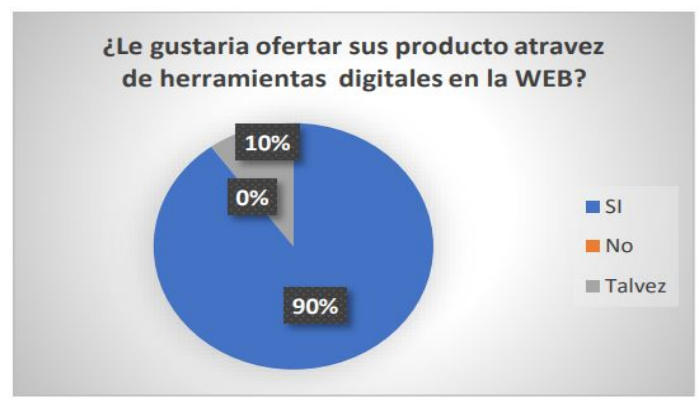

Figura 8. Porcentaje de preferencia de uso a futuro de herramientas digitales en la Web.

Fuente: Elaboración Propia.

En cuanto a microempresarios entrevistadas que publicitan sus productos/servicios utilizando herramientas digitales a través de redes sociales, se les pregunto si estarían dispuesto en implementación de website para sus microempresas, $89 \%$ respondió afirmativamente, ya que tienen claro que la inversión en tener su propio dominio a futuro le ayudara a incrementar sus ventas, y por ende incrementar su patrimonio. De este grupo el $90 \%$ tiene claro que los sistemas recomendadores ayudaran positivamente a predecir hacia donde esta las preferencias y gusto de sus clientes, y por ende a mejorar la toma decisiones.

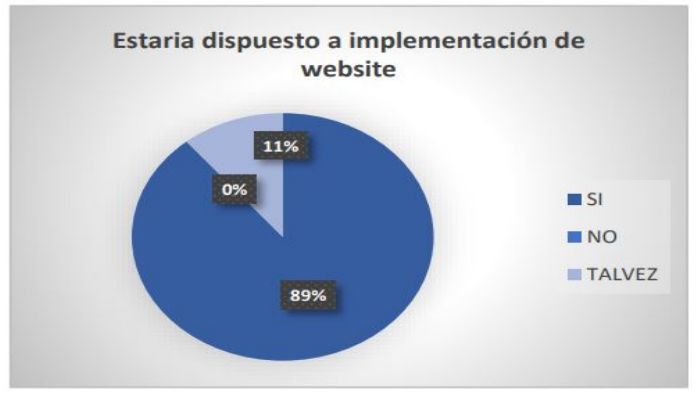

Figura 9. Porcentaje de preferencia de uso a futuro de herramientas digitales en la Web.

Fuente: Elaboración Propia.

\section{Discusión}

De acuerdo a los resultados que se obtuvieron en la investigación es necesario establecer ciertos aspectos muy relevantes del mismo, como: en los últimos años en Ecuador con incremento del uso del internet, uso de la tecnología móvil (smartphone), herramientas digitales y el e-commerce, han ayudado a las empresas y microempresas a publicitar sus productos/servicios. De acuerdo a expertos, en el aérea el boom tecnológico en estos momentos son los sistemas recomendadores, que los usuarios finales a través de sus 


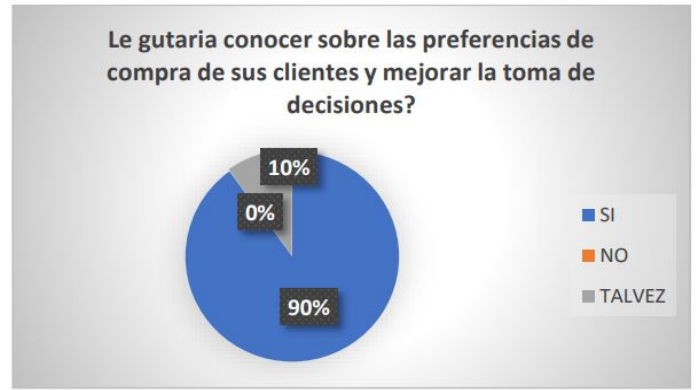

Figura 10. Porcentaje de preferencia en compras por clientes.

Fuente: Elaboración Propia.

fanPage, grupos en WhatsApp, Twitter, entre otros, les ha permitido conocer cuáles son las características y gustos de sus clientes activos. Que la tendencia a crear sus propios website es positiva, ya que aumentado su contratación y que observan como los microempresarios invierten en estos servicios; aunque muchos no tienen conocimientos técnicos en sistemas de recomendación y tratamiento de información, saben que esto es la tendencia y es lo que desean.

En la actualidad ante el manejo de grandes volúmenes de información en bases de datos es sin estructurar en la red, se están desarrollando nuevos algoritmos, formas de almacenar, organizar, buscar, clasificar, etc. Los expertos acotan que esta manera de manipular y clasificar grandes volúmenes de información en la web ha dado origen a los sistemas recomendadores, es importante escoger un buen sistema de filtrado; y que es necesario que los microempresarios le den la importancia y la credibilidad de adquirir esta tecnología, ayudándoles a tener ventajas competitivas y aumentar sus ventas.

\section{CONCLUSIONES}

La manipulación de los grandes volúmenes de datos es un reto para microempresas, pero también será una gran oportunidad para otras. Escoger un buen sistema de filtrado permitirá tener mayor conocimiento de las tendencias y gustos de los usuarios. La calidad y cantidad de los datos e informaciones es más importante que los algoritmos. Los microempresarios están dispuestos en un alto porcentaje adquirir un sistema recomendador ya que es una herramienta que le permitirá mejorar la gestión de la información en su organización. Crear un algoritmo recomendador que se ajuste al contexto de las PYMES en función de su calidad informativa, optimo sin riesgos de perdidas, sin invertir demasiado dinero y esfuerzo, facilitara la toma de decisiones, permitiendo a los microempresarios incrementar su nivel de confianza y credibilidad en el uso del internet para la comercialización de sus productos/servicios a través de los Website.

\section{Agradecimientos}

Nuestro profundo agradecimiento a los microempresarios de la ciudad de Babahoyo y Guayaquil; a los expertos en marketing digital y desarrolladores de Website, por toda la información y apoyo brindado para que el presente proyecto de investigación tenga los resultados que se propusieron desde el inicio, es verdad que falta mucho por hacer, pero consideramos que es el inicio de muchos estudios que se van a emprender desde hoy en adelante. A los expertos en comunidades de la ciudad de Guayaquil, especialmente a los de la empresa Expert Consulting por haber compartido con nosotros sus valiosas experiencias en el área de e-commerce. A los estudiantes de noveno de Ingeniería en sistemas de la Facultad de Administración Finanzas e Informática por su valioso aporte en la recopilación, tabulación de los datos.

\section{REFERENCIAS BIBLIOGRÁFICAS}

ARCOTEL (2017). 46,4\% de usuarios del Servicio Móvil Avanzado poseen un smartphone - Agencia de Regulación y Control de las Telecomunicaciones - Ecuador.

Carrillo Agurto, J. J. (2017). Implementación de un sistema de información para mejorar la gestión de los procesos de compra, venta y almacén de productos deportivos en la tienda casa de deportes rojitas eir ltda.-chimbote; 2014.

Ekos (2016). EKOS NEGOCIOS - Detalle Artículo.

El Comercio (2016). Las ventas on line amplían el mercado - Revista Líderes.

El Telégrafo (2016). El Telégrafo - Menos del $20 \%$ de empresas realiza comercio electrónico.

Galán Nieto, S. M. (2007). Filtrado Colaborativo y Sistemas de Recomendación.

Group Miniwatts Marketing (2017). Internet World Stats Usage and Population Statistics.

Herlocker, J. L., Konstan, J. A., Borchers, A., and Riedl, J. (1999). An algorithmic framework for performing collaborative filtering. In Proceedings of the 22Nd Annual International ACM SIGIR Conference on Research and Development in Information Retrieval, SIGIR '99, pages 230-237, New York, NY, USA. ACM.

Herlocker, J. L., Konstan, J. A., Terveen, L. G., and Riedl, J. T. (2004). Evaluating collaborative filtering recommender systems. ACM Trans. Inf. Syst., 22(1):5-53.

Herrera-Viedna, E., Porcel, C., and Hidalgo, L. (2004). Hipertext.net: Revista Académica sobre Documentación Digital y Comunicación Interactiva.

IAB (2014). IAB - Ecuador.

INEC (2015). Nothing found for Documentos Web-inec Estadisticas_Economicas DirectorioEmpresas Directorio_Emp.

López, J. F., Valdéz, E. N., Cueva, J., Sanjuán, O., Pelayo, B., and Montenegro, C. (2011). Plataforma de recomendación de contenidos para libros electrónicos inteligentes basadas en el comportamiento de los usuarios. TECCIENCIA, 6(11):30-44.

Rangel, J. R. (2014). Minería de datos como soporte a la toma de decisiones empresariales en una arquitectura Soa. Innovación, Ingeniería y Desarrollo, 1(1).

Robin, B. (2002). Hybrid Recommender Systems: Survey and Experiments. User Modeling and User-Adapted Interaction, 12(4):331-370.

Sanjuan-Martinez, O., G-Bustelo, B. C. P., Crespo, R. G., and Franco, E. T. (2009). Using recommendation system for elearning environments at degree level. International Journal of Interactive Multimedia and Artificial Intelligence, 1(2). 
Sarwar, B., Karypis, G., Konstan, J., and Riedl, J. (2017). ItemBased Collaborative Filtering Recommendation Algorithms. 